\title{
Exemplar Training and A Derived Transformation of Functions in Accordance with Symmetry and Equivalence
}

Article in The Psychological record · January 2010

DOI: 10.1007/BF03395577 · Source: OAI

CITATIONS

23

5 authors, including:

Serafín Gómez

Universidad de Almería

3 PUBLICATIONS 43 CITATIONS

SEE PROFILE

Yvonne Barnes-Holmes

Ghent University

169 PUBLICATIONS 6,423 CITATIONS

SEE PROFILE
READS

125

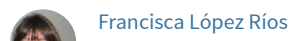

Universidad de Almería

25 PUBLICATIONS 415 CITATIONS

SEE PROFILE

Some of the authors of this publication are also working on these related projects:

Odysseus View project 


\title{
EXEMPLAR TRAINING AND A DERIVED TRANSFORMATION OF FUNCTIONS IN ACCORDANCE WITH SYMMETRY AND EQUIVALENCE
}

\author{
SERAFÍN GÓMEZ, FRANCISCA LÓPEZ, \\ and CARMEN BAÑOS MARTÍN \\ University of Almería. Spain
}

\section{YVONNE BARNES-HOLMES and DERMOT BARNES-HOLMES National University of Ireland, Maynooth, Ireland}

\begin{abstract}
The current study consisted of 2 parts, with the same 4 normally developing 4-yr-old children employed across both parts. The primary aim of Part 1 was to replicate previous research on exemplar training and its impact upon the emergence of repertoires of derived symmetry or mutually entailed relations. In this part of the study, the children were trained in action-object conditional discriminations and were then exposed to a symmetry test for the derived object-action relations. All 4 participants demonstrated derived symmetry without needing exemplar training. In Part 2, the conditional discrimination training was extended such that 2 actions were associated with each object and the children were then required to derive the target equivalence relations between the 2 related actions (i.e., actionaction equivalence). Once again, a multiple baseline design was employed for the systematic introduction of exemplars of actionaction training. The results from Part 2 indicated that 3 of the 4 children demonstrated the target-derived equivalence relations only after they received explicit equivalence training. These findings support the role given to exemplar training within a Relational Frame Theory interpretation of derived stimulus relations and suggest its utility as a means of establishing symmetry and equivalence when they are found to be absent.
\end{abstract}

Numerous researchers in the last decade have suggested that Relational Frame Theory (RFT) offers a coherent account of established

This research was conducted as part of Carmen Baños Martin's predoctoral research program under the supervision of Serafín Gómez Martín and Francisca López Ríos. The research was in part supported by the Grupo de Análisis Clínico y Experimental en Psicología. We thank Colegio de Educación Infantil y Primaria Virgen de la Paz for the facilities given to conduct the experiments reported here. Portions of these data were presented at the Symposium entitled RFT, Contextualism and Education at the World Conference on ACT, RFT and The New Behavioral Psychology, Linköping, Sweden. Correspondence concerning this article should be addressed to Serafín Gómez Martín, Departamento de Personalidad, Evaluación y Tratamiento Psicológicos, Facultad de Humanidades, Universidad de Almería, 04120, Almería, Spain. (E-mail: sgomez@ual.es). 
behavioral effects, including stimulus equivalence and other derived relations of comparison and opposition (Dymond \& Barnes, 1995; Hayes, Barnes-Holmes, \& Roche, 2001). Although numerous such effects are now well established in the behavioral literature (e.g., Hayes et al., 2001; Sidman, 1994), some authors have raised questions concerning the types of histories necessary to generate repertoires of relational responding (e.g., Boelens, 1994; Galizio, 2003; Horne \& Lowe, 1996; Palmer, 2004). According to RFT, one of the most effective means of establishing relational repertoires involves explicit training in exemplars of the target relational skill. For example, if one wishes to establish repertoires of symmetry or mutually entailed relations of coordination, then one would explicitly train a symmetry response on a particular exemplar and then test for the derived symmetry response on a new untrained exemplar. According to RFT, such a recursive pattern of training and testing across exemplars (referred to as multiple exemplar training) constitutes a powerful tool for establishing relational skills such as symmetry (see Barnes, 1994, 1996; Barnes \& Holmes, 1991; Barnes \& Roche, 1996; Hayes, 1991, 1994; Hayes \& Hayes, 1989) or other derived relational repertoires (e.g., Gómez, Barnes-Holmes, \& Luciano, 2001, 2002).

In the language of RFT, derived relational responses (i.e., relational frames) are generalized operant response classes established by a history of reinforcement across exemplars. Although numerous such relational repertoires can be distinguished and have been studied empirically, all relational frames are believed to possess the three generic properties of mutual entailment, combinatorial entailment, and the transfer/ transformation of stimulus functions (e.g., Hayes, 1991, 1994; Hayes \& Hayes, 1989, 1992). According to the simple concept of mutual entailment (similar to Sidman's concept of symmetry, but broader in scope given the fact that it can accommodate other relations such as comparison, difference etc.), a history of explicitly reinforced A-B responding will generate derived mutually entailed B-A relations. According to the more extensive concept of combinatorial entailment, a history of explicitly reinforced $A-B$ and $A-C$ responding will generate derived combinatorially entailed $A-C$ and $C-A$ relations. Finally, a transfer of stimulus function involves a derived relation between stimuli and a transformation of function in one stimulus based on its relation to another. For example, if $A$ and $B$ participate in the mutually entailed relation of symmetry or coordination, and an eliciting function is established for $\mathrm{B}$, the eliciting function may be transferred to $\mathrm{A}$ via the derived relation between the two stimuli and $A$ would acquire the same function as $B$. However, consider now if $A$ and $B$ participate in a mutually entailed relation of more-than where $A$ is more than $B$. If a function is then established for $B$ (e.g., pressing a key on the keyboard twice) then $A$ would acquire an even greater function than $B$ (e.g., a subject would press the key more than twice) based on the precise comparative relation that exists between the two stimuli.

In spite of a now considerable body of evidence demonstrating different and complex patterns of derived relational responding (e.g., 
Dymond \& Barnes, 1995, 1996; Roche \& Barnes, 1996, 1997; Steele \& Hayes, 1991; Wulfert \& Hayes, 1988), there have been many fewer studies concerned with the specific histories necessary to establish or facilitate these skills when they are found to be absent (e.g., BarnesHolmes, Barnes-Holmes, Roche, \& Smeets, 2001a, 2001b).

In the first study by Barnes-Holmes et al. (2001a) the researchers attempted to determine whether exemplar training would facilitate transformations of function in accordance with symmetry. Sixteen children, between 4 and 5 years old, were employed across four experiments. The basic experimental format first established/facilitated naming repertoires for all of the experimental stimuli. The children were then trained in actionobject conditional discriminations (e.g., experimenter waves-child selects toy car) and were thereafter tested for derived object-action symmetry relations (e.g., experimenter presents toy car-child waves). Across several experimental sessions, a multiple-baseline design was used to introduce exemplars of explicit symmetry training for children who failed the symmetry test. In total, 13 of the 16 participants failed to demonstrate derived object-action (Experiments 1-3) or action-object (Experiment 4) symmetry until they received explicit symmetry training.

In a subsequent series of almost identical studies, the same researchers (Barnes-Holmes et al., 2001b) investigated whether the same facilitative effects of exemplar training in symmetry would be observed in the absence of explicit name training. In this study, a further 16 children, again aged between 4 and 5 years old, were employed across three experiments. Across Experiments 1 and 2, none of the 8 children showed derived object-action (Experiment 1) or action-object (Experiment 2) symmetry until they received explicit symmetry training. In Experiment 3, a further 4 children also demonstrated derived symmetry only after receiving exemplars of symmetry training, in spite of prior exposure to object-action pretraining. Taken together, these results lend considerable support to the RFT view that exemplar training constitutes a powerful tool for the facilitation/establishement of repertoires of derived symmetry or responding in accordance with mutually entailed relations of coordination.

Although a clear facilitative effect for the exemplars of symmetry training was demonstrated, one important question raised by the two previous studies concerned the very limited number of exemplars required (i.e., only one or two) before the children produced the target symmetry responses. The limited number of exemplars needed to demonstrate the skills might reflect that derived symmetry already existed in the behavioral repertoires of the children in question. Indeed, the authors argued that the experimental work almost certainly did not establish a repertoire of symmetry responding $a b$ initio, but that the exemplar training did establish the experimental context as a cue for demonstrating the preexperimentally established symmetry repertoire. In samples of normally developing children between the ages of 4 and 5 years old, it is perhaps inevitable that repertoires of derived symmetry will be preexisting and that the existing experimental preparation can only serve to extend these repertoires to novel stimuli and/or tasks. 
A very recent series of RFT studies, however, do appear to have more effectively addressed the issue of establishing relational repertoires ab initio. In an analysis of relational responding in accordance with morethan and less-than, Barnes-Holmes, Barnes-Holmes, Smeets, Strand, \& Friman (2004) reported "the first attempt to generate repertoires of relational responding, as generalized operant behaviors, when they are found to be absent in young children" (p. 531) using exemplar training. Three children, between 4 and 6 years old, were exposed to a basic problem-solving task that involved two or three identically sized (hence mutually and combinatorially entalied arbitrary relations) paper coins. On each trial, the experimenter described how the coins were related in terms of value and the child was required to pick the coin(s) that bought "as many sweets as possible." The results of the study indicated that all 3 children failed baseline tests of the arbitrary more and less relations among arrays of four coins. However, interventions suggested by RFT, including exemplar training across stimulus sets and different numbers of coins successfully established highly complex and flexible patterns of more and less responding in all 3 children.

In a very similar study, some of the same researchers (BarnesHolmes, Barnes-Holmes, \& Smeets, 2004) reported the first attempt to generate repertoires of relational responding in accordance with opposite, as generalized operant behavior, when they are found to be absent for another 3 children between 4 and 6 years old. On each experimental trial, the child was presented once again with an array of identically sized paper coins and relations of opposition among the coins were specified. For example, the child may have been instructed as follows: "This coin (e.g., A) buys many (or few) sweets, and is opposite to this coin (B), which would you take to buy as many sweets as possible"? Once again, all 3 children failed baseline tests for specific patterns of relational responding in accordance with opposite involving four coins. However, explicit training across exemplars, including different stimuli and different numbers of stimuli established complex patterns of opposite responding for all 3 children. In summary, therefore, there appears to be considerable evidence of the utility of exemplar training for the facilitation and establishment of different repertoires of relational responding.

The present study had two goals. The first goal was to attempt to partially replicate the work of Barnes-Holmes et al. (2001a, Experiment 2 ) in terms of investigating the emergence of repertoires of derived mutually entailed symmetry relations and the utility of exemplar training in this regard, once again with a sample of young children (referred to herein as Part 1). The reader should note, that for ease of communication the term symmetry and equivalence will sometimes be used instead of transformation of functions in accordance with symmetry or equivalence (see Barnes, 1996, for a detailed discussion of why the latter terms are more accurate from a relational frame perspective). The second aim of the current work was to extend the original study by examining combinatorially entailed equivalence relations and the utility of exemplar training in this context (referred to herein as Part 2). 
In order to achieve the two key experimental objectives, the current work employed a methodology that was almost identical to that used in the two previous symmetry studies by Barnes-Holmes and colleagues (2001a, 2001b). Four 4- to 5-yr-old children were exposed to the methodology across two experimental parts. In Part 1, children were trained to name two actions and two objects by demonstrating listening, echoic, and tacting behaviors. They were then trained in action-object conditional discriminations using the previously named actions and objects (e.g., when the experimenter waved, choosing a toy car was reinforced, and when the experimenter clapped, choosing a doll was reinforced). After the training, the children were exposed to a symmetry test for derived objectaction relations (e.g., would the children wave when a car was presented and clap when a doll was presented?). If they failed the symmetry test a multiple-baseline design would be used to introduce exemplar training (i.e., explicit symmetry training). In Part 2, the children were trained in an action-object conditional discrimination (e.g., waving-A1 and clappingA2). They were then trained to name two novel actions demonstrating listening, echoic and tacting behaviors (e.g., touching shoulder-touching forehead). The children were then trained in a second action-object conditional discrimination using the two novel actions just learned and the same objects (e.g., touching forehead-A1 and touching shoulderA2). Subsequently, they were exposed to a symmetry object-action test using the novel actions learned. If the children passed the symmetry test, they were exposed to an equivalence action-action test (e.g., touching shoulder-waving and touching forehead-clapping). If they failed the equivalence test, across subsequent sessions a multiple-baseline design was used to introduce exemplar training (i.e., explicit equivalence training) for those participants who failed the equivalence test.

\section{General Method}

\section{Participants}

Four children, two males (P2 and P4) and two females (P1 and P3), aged between 4:6 and 4:10 years/months old, all participated in both Parts 1 and 2 of the current study. The gender and age profiles of each child are provided in Table 1. The children were enrolled in "Primary One" of a local public school in Almería (Spain) and were selected by their teachers on the basis that neither teachers nor parents had identified them as presenting any form of learning difficulty.

Table 1

Participant Gender and Age Profiles

\begin{tabular}{ccc}
\hline Participant & Gender & $\begin{array}{c}\text { Age } \\
\text { (Yr:Mth) }\end{array}$ \\
\hline 1 & Female & $4: 10$ \\
2 & Male & $4: 6$ \\
3 & Female & $4: 10$ \\
4 & Male & $4: 8$ \\
\hline
\end{tabular}




\section{Setting and Apparatus}

All experimental trials were conducted in a vacant school classroom in the children's school. All experimental trials were conducted using one desk and two chairs, with the chairs facing each other across the desk. During the experimental trials, each participant sat on one chair on one side of the desk facing the experimenter. Participants completed all of the experimental trials individually.

All of the stimuli employed in the current experiment consisted of common items familiar to young children (e.g., a story book and a doll) and a series of simple motor sequences (e.g., waving hands and touching feet). The experimental stimuli and actions employed in Parts 1 and 2 of the experiment are presented in Tables 2 and 3, respectively. For experimental purposes, all stimuli were referred to using alphanumeric labels (e.g., the toy car was referred to as A1 and the doll as A2) that enabled them to be organized into various stimulus classes that consisted of specifically trained conditional discriminations (e.g., wave-A1) and to-be-derived symmetry (e.g., A1-wave) and equivalence relations. Participants were not informed of these labels or class organization. A range of additional materials were employed as reinforcers, including a small tray, colored beads, and a marked glass jar. These stimuli had been deemed "reinforcers" based upon preexperimental interactions with the children involved.

Table 2

Stimuli and Actions Employed in Each Session of Part 1 of the Experiment

\begin{tabular}{ccc}
\hline Session No & Stimuli & Actions \\
\hline I & Doll (A1) & Waving \\
& Car (A2) & Clapping \\
II & Story Book (B1) & Arms out \\
& Flower (B2) & Arms in \\
\hline
\end{tabular}

Table 3

Stimuli and Actions Employed in Each Session of Part 2 of the Experiment

\begin{tabular}{cll}
\hline Session No & Stimuli & \multicolumn{1}{c}{ Actions } \\
\hline I & Doll (A1) & Waving \\
& Car (A2) & Clapping \\
& & Touching forehead \\
& Touching shoulder \\
II & Story Book (B1) & Arms out \\
& Flower (B2) & Arms in \\
& & Touching waist \\
III & Touching neck \\
& Teddy Bear (C1) & Touching nose \\
& Pencil Sharpener (C2) & Touching ear \\
IV & & Scratching arm \\
& & Scratching face \\
& Cup (D1) & Rubbing head \\
& Spoon (D2) & Scratching tummy \\
& & Touching knee \\
& & Touching elbow \\
\hline
\end{tabular}




\section{Experimental Design}

The current study consisted of two parts (referred to as Parts 1 and 2 , and conducted in that order). The basic procedure employed across both parts of the study consisted of the training of a series of action-object conditional discriminations and thereafter testing the derived symmetry relations (Part 1) or the derived equivalence relations (Part 2). Both parts of the study also contained a phase of explicit training of the test performance that was employed as a form of exemplar training across stimulus sets for those children who failed the symmetry or equivalence test. In Part 1 of the study, the 4 children were trained in two simple action-object discriminations and were thereafter tested on the derived symmetrical object-action relations. For those children who failed the first symmetry test, a multiple baseline design determined whether they would be reexposed to the conditional discrimination training or whether they would receive explicit object-action (symmetry training). In both cases, the children then proceeded directly to a second set of conditional discriminations on which the impact of the previous training on the second symmetry test could be determined. In Part 2 of the study, the conditional discrimination training was extended and the children were now trained to conduct two actions in the presence of each of two objects (e.g., wave/touch forehead-A1 and clap/touch shoulder-A2) and were then tested on the derived action-action equivalence relations (e.g., wave-touch forehead). Once again, for those children who failed the first equivalence test, a multiple baseline design determined whether they would be reexposed to the conditional discrimination training or whether they would receive explicit action-action (equivalence training). In both cases, the children then proceeded directly to a second set of conditional discriminations on which the impact of the previous training on the second equivalence test could be determined. Exemplar training continued across multiple stimulus sets until all children passed the equivalence test.

\section{General Procedure}

All experimental sessions conducted with the children lasted between 5 and 25 minutes. Participants were exposed to one session per day, with a break of $5 \mathrm{~min}$ (the children were allowed to play in an adjacent room during this break). Between phases each child participated in the next session on the next weekday (availability permitting). Each session in Part 1 contained three or four experimental phases and all 4 children completed Part 1 in a maximum of only two sessions. Participation in Part 2 was longer. Each session in this part of the study contained five or six phases. Nonetheless, all 4 children completed Part 2 in a maximum of four sessions.

\section{Programmed Consequences}

At the beginning of each session, the experimenter placed the bead tray and beads on the table and participants were instructed that they were going to play a game in which a bead would be awarded for each correct 
response and that beads could then be exchanged for a preselected picture when the mark on the glass jar (50 beads) had been reached (see Smeets, Barnes, \& Luciano, 1995). Correct responses during all training trials were followed by the words "Yes, you are correct. Good boy/girl. Take a bead." Incorrect responses during training were followed by the experimenter saying: "No, this is not right. No bead." No beads could be selected after an incorrect response had been emitted. No programmed consequences followed any test trial.

\section{Interobserver Reliability}

Twenty-five percent of training trials and all testing trials across Parts 1 and 2 of the experiment were observed by an independent observer who had no knowledge of experimental psychology. The observer could not see the experimenter's data sheets during the experimental sessions. Both the observer and the experimenter recorded the children's responses, in terms of the actions they engaged in or the objects they selected, and they scored each response as either correct or incorrect. Agreement between the observer's and experimenter's recording was $100 \%$.

\section{Part 1}

\section{Exemplar Training and a Derived Transformation of Functions in Accordance With Symmetry}

\section{Method}

\section{Session 1}

Phase1. Name training. In Phase 1 of Session I, all participants were explicitly trained to emit the names of two gross motor activities, waving and clapping, and two objects, A1 and A2 (i.e., a doll and a toy car, respectively). In order to ensure that all of the actions and objects used for the experimental trials could be readily discriminated, the name training of all of these stimuli involved explicitly reinforcing appropriate listening, echoic, and tacting behaviors. For objects, this involved reinforcing the choice of an object given its name (listening); reinforcing the spoken name of the object when asked, for example "Say car" (echoic behavior); and reinforcing the spoken name when asked "What is this?" (tacting). Specifically, on an object-listening trial, stimuli A1 and A2 were placed on the table, and the child was asked, for example "Can you point to (A1) (i.e., the doll)?" A correct listening response involved the child pointing the correct object (i.e., A1). On an object-echoic trial, the experimenter asked, for example, "Can you say car?" and a correct echoic response involved the child repeating the word "car" or any phrase containing this word. On an object-tacting trial, the experimenter pointed, for example, to A1 and the child was asked "What is this?" A correct tact response involved the child saying the appropriate name (i.e., doll). The same procedure was adopted for training, listening, echoic, and tacting 
behaviors with respect to A2. Each participant was exposed to the three trial-types of explicit name training of each of the two objects in a block of 24 quasi-random training trials, with four exposures to each trial-type. The object name training continued until each participant reached a criterion of 24 consecutive object naming trials, after which it was assumed that the appropriate naming repertoires were established. Participants then proceeded to explicit name training with the experimental actions.

The explicit name training of the actions in Phase 1 was almost identical to the name training of the objects. Specifically, during a wavinglistening trial, the child was asked "Show me waving" and a correct response consisted of the child demonstrating the waving action. During a waving-echoic trial, the experimenter asked, "Can you say waving?" and a correct echoic response consisted of the child repeating the word "waving" back to the experimenter within $3 \mathrm{~s}$. During a waving-tacting trial, the experimenter waved her arm in a left-right-left sequence at the child while asking "What I am doing?" A correct tact response consisted of the child saying "waving" or any phrase containing the word "waving." Listening, echoic, and tacting trial-types were similarly conducted for the clapping action. Once again, each participant was exposed to the three trial-types of explicit name training of each of the two actions in a block of 24 quasi-random training trials, with four exposures to each trial-type. When participants responded correctly on 24 consecutive action naming trials it was assumed that the appropriate naming repertoires were established. In this way, the name training in Phase 1 consisted of at least two blocks of 24 trials (minimum of 48 training trials), after which participants proceeded to Phase 2.

Phase 2: Action-object training. Following the name training, participants were introduced to the action-object conditional discrimination training. During the first four action-object training trials, stimuli A1 and A2 were placed beside one another on the table (the left-right positions of these stimuli were randomized across trials). The instructions were as follows: "When I wave/clap at you, I want you to pick the doll (A1)/the car (A2). I will tell you if you have chosen the right or wrong one." The same procedure was then used for all subsequent Phase 2 training trials, except that the verbal instruction was omitted after the first four trials. Reinforcement was provided for selecting $A 1$ in the presence of the experimenter waving (i.e., explicitly training the relation waving-A1) and A2 in the presence of the experimenter clapping (i.e., explicitly training the relation clapping-A2). The Phase 2 conditional discrimination was presented in blocks of eight quasi-random training trials, with the A1 and A2 trial-types each presented four times. Training continued until each participant reached a criterion of eight consecutively correct responses trials, after which it was assumed that the appropriate action-object relations were established. Participants then proceeded to Phase 3.

Phase 3: Test for derived symmetrical object-actions relations. Phase 3 consisted of a test for the derived symmetrical object-actions relations based on the action-object training in Phase 2. During each Phase 3 trial, 
either A1 (or A2) was placed in the center of the table and the child was required to emit the appropriate action in the presence of each object (e.g., wave in the presence of $\mathrm{A} 1$ ) within a 10-s interval. The experimenter then remained silent and looked directly down at the near edge of the table, so that the participant could not see the experimenter's face. The experimenter looked up only when the child emitted a response. The experimenter was instructed about the critical importance of doing this appropriately and avoiding in this way giving the child any cue about the correct response. The target derived relations for Phase 3 were A1-waving and A2-clapping. Hence, if a participant failed to clap or wave appropriately during the interval, the trial was recorded as incorrect. The next trial then commenced immediately and the alternative A stimulus was presented.

All Phase 3 trials were presented within a block of eight quasi-random test trials that consisted of four exposures to two trial-types, one for the derived symmetrical A1-waving relation and one for the A2-clapping relation. In order to pass the test, participants were required to reach a criterion of eight consecutively correct responses (100\% correct), after which it was assumed that the derived action-object relations were established. If, however, participants did not pass the test on the first exposure, the multiple-baseline design across participants determined whether they were reexposed to Phase 2 (i.e., the action-object training) or whether they proceeded to Phase 4. According to the multiple baseline design, if P1 failed the test she was exposed immediately to Phase 4, and the remaining 3 participants were reexposed to Phase 2. That is, the first participant (P1) was introduced to the Phase 4 explicit symmetry training immediately after the first failure on the derived object-action test in Phase 3 (and thus would not be reexposed to Phase 2). In contrast, the second participant (P2) would be reexposed to Phases 2 and 3 after the first failure in Phase 3 and would then only be exposed to Phase 4 after a second failure in Phase 3 . The third participant (P3) would be reexposed to Phase 2 twice (if necessary) and would only proceed to Phase 4 after three failures on Phase 3. Finally, P4 would be reexposed to Phase 2 three times (if necessary) and would only proceed to Phase 4 after four failures on Phase 3. In this way, the impact of the Phase 4 training could be compared directly with systematic reexposures to Phases 2 and 3.

Phase 4: Explicit object-action (symmetry) training. Phase 4 was almost identical to Phase 3 , except that programmed consequences were delivered after each response, or at the end of the 10-s interval if no response occurred. In other words, the target object-actions relations (A1waving and A2- clapping) were explicitly trained (rather than tested). Note, however, that no instructions were provided during this phase, (i.e., the child was not told what to do at the beginning of a trial). Phase 4 training continued until each participant had emitted eight consecutively correct responses. Once again, the trials were presented in blocks of eight quasirandom training trials that consisted of four exposures to each of the two A1 and A2 trial-types. Exposure to the explicit training in Phase 4 of Session 1 constituted the first exemplar in symmetry training and marked the end of Session I. All participants thereafter proceeded directly to Session II. 


\section{Session II}

Session II was almost identical to Session I, except that novel stimuli and actions, and their respective names were employed (see Tables 2 and 4). Specifically, the action waving was replaced with Arms Out and clapping was replaced with Arms In. A1 was replaced with B1 (i.e., story book) and A2 was replaced with B2 (i.e., flower). Thus, the action-object relations arms out-B1 and arms in-B2 were trained explicitly in Phase 2 and the derived object-action relations (B1-arms out and B2-arms in) were tested in Phase 3. Once again, if a participant passed the symmetry test in Phase 3 of Session II, her/his participation in the experiment was terminated for the time being. Alternatively, the multiple baseline design once again determined which participants would proceed immediately to Phase 4, which would be reexposed to Phases 2 and 3 , and exactly how many reexposures to Phases 2 and 3 would be conducted. Exposure to the explicit training in Phase 4 of Session II constituted the second exemplar in symmetry training and all participants thereafter proceeded directly to Part 2 of the study.

Table 4

Relations Trained and Tested in Each Part of the Experiment

\begin{tabular}{|c|c|c|}
\hline $\begin{array}{l}\text { Session } \\
\text { No. }\end{array}$ & Trained Action-Object Relations & $\begin{array}{l}\text { Tested Object-Action Symmetry Relations (Part 1) } \\
\text { \& Action-Action Equivalence Relations (Part 2) }\end{array}$ \\
\hline \multicolumn{3}{|c|}{ Part 1} \\
\hline I & Wave-A1 \& Clap-A2 & A1-Wave \& A2-Clap \\
\hline$\|$ & Arms Out-B1 \& Arms in-B2 & B1-Arms Out \& B2-Arms in \\
\hline \multicolumn{3}{|c|}{ Part 2} \\
\hline I & $\begin{array}{l}\text { Wave-A1 \& Clap-A2 } \\
\text { Touch forehead-A1 \& Touch shoulder-A2 }\end{array}$ & $\begin{array}{l}\text { A1-Touch forehead \& A2-Touch shoulder } \\
\text { Touch forehead-wave \& Touch shoulder-clap }\end{array}$ \\
\hline$\|$ & $\begin{array}{l}\text { Arms out-B1 \& Arms in-B2 } \\
\text { Touch waist-B1 \& Touch neck-B2 }\end{array}$ & $\begin{array}{l}\text { B1-Touch waist \& B2-Touch neck } \\
\text { Touch waist-Arm out \& Touch neck-Arm in }\end{array}$ \\
\hline III & $\begin{array}{l}\text { Touch nose-C1 \& Touch ear-C2 } \\
\text { Scratch arm-C1 \& Scratch face C2 }\end{array}$ & $\begin{array}{l}\text { C1-Scratch arm \& C2-Scratch face } \\
\text { Scratch arm-Touch nose \& Scratch face-Touch ear }\end{array}$ \\
\hline IV & $\begin{array}{l}\text { Rub head-D1 \& Scratch tummy-D2 } \\
\text { Touch Knee-D1 \& Touch elbow-D2 }\end{array}$ & $\begin{array}{l}\text { D1-Touch Knee \& D2-Touch elbow } \\
\text { Touch knee-Rub head \& Touch elbow-Scratch tummy }\end{array}$ \\
\hline
\end{tabular}

\section{Results of Part 1}

The data recorded with all 4 participants during each of the four training or test phases of Sessions I and II in Part 1 are presented in Table 5. For illustrative purposes, consider the results obtained with P2. Participant 2 completed the name training with waving and clapping and $A 1$ and $A 2$ in 49 trials, followed by only 15 trials of the action-object conditional discrimination training. He failed the first symmetry test in Session I with six out of eight correct responses and was thus reexposed to Phase 2, which was completed in the minimum number of trials. In Session II, he also completed the name training in the minimum number 
of trials and thereafter required only 10 training trials to complete the action-object conditional discrimination training with arms out and arms in and B1 and B2. He passed the second exposure to the symmetry test in Session II immediately, thus requiring no exposure to explicit symmetry training (Phase 4).

Table 5

Number of Training Trials Required and Correct Responses During Test Trials for Each Participant in Part 1 of the Experiment

\begin{tabular}{lcccc}
\hline Participant & 1 & 2 & 3 & 4 \\
\hline Session I & 51 & 49 & 48 & 48 \\
Phase 1: Name training & 12 & 15 & 12 & 16 \\
Phase 2: Action-object training & 12 & $8 / 8$ & $5 / 8$ \\
Phase 3: Symm. Test (object-action) & $8 / 8$ & $--^{*}$ & - & \\
Phase 4: Object-action training & - & & & 48 \\
Session II & - & 48 & - & 12 \\
Phase 1: Name training & - & 10 & - & $8 / 8$ \\
Phase 2: Action-object training & - & $8 / 8$ & - & \\
Phase 3: Symm. Test (object-action) & - &
\end{tabular}

Note. ${ }^{*}$ indicates that the participant was reexposed to action-object training. In all cases, participant completed the training in eight trials (i.e., the minimum number required).

In summary, therefore, 2 of the children (P1 and $\mathrm{P} 3$ ) passed the symmetry test on their first exposure in Session I. In accordance with the multiple baseline design, P2 and P4 were reexposed to Phase 2, rather than proceeding to Phase 4 . These 2 participants then immediately passed the second symmetry test in Session II without first receiving explicit symmetry training. It was therefore unnecessary to introduce exposure to the Phase 4 explicit symmetry training through the multiplebaseline design across participants as was originally planned.

\section{Part 2}

\section{Exemplar Training and a Derived Transformation of Functions in Accordance with Equivalence}

The second goal of the present study was to determine whether exemplar training would facilitate the transformation of function in accordance with equivalence (rather than symmetry as happened in the first part of this study) with the same children from Part 1. The results from Part 1 indicated that all 4 children were capable of perfect or relatively strong performances on their first exposure to the test for the derived symmetrical object-action relations, without the need for explicit symmetry training. According to RFT, these abilities may be described as a transformation of functions in accordance with symmetry involving mutually entailed object/action relations. In some sense, the results from Experiment 1 were not entirely surprising given that the children were presented with a test involving relatively simple transformations of symmetry functions with only mutually entailed relations. According to this 
view, a more difficult test would involve combinatorially entailed relations. Thus, the aim of Part 2 was to determine whether explicit training of the target relations would remediate observed deficits in deriving combinatorially entailed relations. That is, for example would participants demonstrate the target transformations of function in the context of combinatorially entailed relations of coordination (or equivalence)? And if not, could these performances be remediated across exemplars of explicit equivalence training. Specifically, in Part 2 participants were trained in two action-object conditional discriminations involving four actions and only two objects (e.g., Action 1-Object 1, Action 2-Object 2; Action 3Object 1, Action 4-Object 2) and then tested for the derived action-action equivalence relations (e.g., Action 3-Action 1 and Action 4-Action 2). For those participants who failed the test initially, a similar multiple baseline design determined reexposure to the conditional discrimination training or to explicit equivalence training across exemplars as alternative means of remediating these deficits in performance.

\section{Method}

Part 2 of the study was very similar in format to Part 1, except that a number of additional methodological features were required in the former in order to include the training and testing of combinatorially entailed equivalence relations as well as mutually entailed symmetry relations. The primary methodological difference between the experimental sessions in Part 2 relative to Part 1 was that the former consisted of seven rather than four experimental phases.

\section{Session I}

Phase1: Action-object training. In Phase 1 of the first session in Part 2, participants were reexposed to the same target action-object relations (waving-doll and clapping-car) from Session I in Part 1. This retraining of the original relations was necessary because they would subsequently be used to form the equivalence relations in the remaining phases of Session I. The action-object training was presented in blocks of eight training trials and participants were again required to reach a mastery criterion of eight consecutively correct responses before proceeding to Phase 2 .

Phase 2: Name training. Phase 2 of Session I in Part 2 was identical to the name training in Phase 1 of Part 1 and involved explicit name training (i.e., listening, echoics, and tacting) with the two novel actions (i.e., touching forehead and touching shoulder). This name training consisted of blocks of 12 trials, two exposures for each of the 3 trials (i.e., listening, echoics, and tacting) for one action (i.e., touching forehead) and two exposures for each of the 3 trials for the other action (i.e., touching shoulder). Participants were required to reach a criterion of 12 consecutively correct responses on the name training before proceeding to Phase 3 .

Phase 3: Action-object training. During Phase 3 of Session I, participants were trained in two new action-object relations between the two novel actions and stimuli A1 and A2 (i.e., touching forehead-A1 and 
touching shoulder-A2). When the novel action-object relations (touching forehead-A1 and touching shoulder-A2) are combined with the original action-object relations (waving-A1 and clapping-A2), then one could derive relations of equivalence or coordination between the actions (i.e., touching forehead-waving and touching shoulder-clapping). Once again, the action-object training was presented in blocks of eight quasi-random trials and participants were required to reach a mastery criterion of eight consecutively correct responses before proceeding to Phase 4 .

Phase 4: Test for derived symmetrical object-actions relations. In Phase 4, a test for the derived object-action symmetry relations (i.e., touching forehead-A1 and touching shoulder-A2) involving the novel action-object relations (i.e., A1-touching forehead and A2-touching shoulder) trained in the previous phase was conducted (the original symmetry relations were presumed to be intact). Once again, the symmetry test comprised a block of eight quasi-random trials with four exposures to the two $A 1$ and $A 2$ trial-types. If participants failed the symmetry test, the multiple baseline design determined whether they would be reexposed to the Phase 3 action-object training or to the explicit object-action (symmetry) training in Phase 5.

According to the multiple baseline design, if $\mathrm{P} 1$ failed the test she would be exposed immediately to Phase 5 , and the remaining 3 participants would be reexposed to Phase 3 . That is, the first participant (P1) would be introduced to the Phase 5 explicit symmetry training immediately after the first failure on the derived object-action test in Phase 4 (and thus would not be reexposed to Phase 3). In contrast, the second participant (P2) would be reexposed to Phase 3 after the first failure in Phase 4 and would then only be exposed to Phase 5 after a second failure in Phase 4. The third participant (P3) would be reexposed to Phase 3 twice (if necessary) and would only proceed to Phase 5 after three failures on Phase 4. Finally, P4 would be reexposed to Phase 3 three times (if necessary) and would only proceed to Phase 5 after four failures on Phase 4 . In this way, the impact of the Phase 5 training could be compared directly with systematic reexposures to Phases 3 and 4 .

Phase 5: Object-action training. Phase 5 was almost identical to Phase 4, except that programmed consequences were delivered after each response, or at the end of the 10-s interval if no response occurred. As in Part 1, the explicit symmetry training continued until each participant had emitted eight consecutively correct responses. Exposure to the explicit training in Phase 5 of Session I would constitute the first exemplar in symmetry training in Part 2.

Phase 6: Test for derived equivalent action-action relations. In Phase 6 , a test for derived equivalent action-action relations was conducted (see Table 4). This test consisted of two trial-types, each of which was presented in a quasi-random order across a block of eight trials. During the action-action test trials, the experimenter, for example, touched her shoulder (or forehead) while silent and looked directly down at the near edge of the table, so that the participant could not see the experimenter's 
face. The experimenter only looked up when the child initiated a response. A 10-s interval was allowed for the child to respond (i.e., clap or wave). If the participant failed to clap or wave during this interval, the trial was recorded as incorrect. If a child passed the equivalence test with eight consecutively correct responses (i.e., touching forehead-waving and touching shoulderclapping), it was assumed that the target equivalence relations had been established, and participation in the experiment was terminated for that child. However, if a child failed the first equivalence test, the multiplebaseline design determined whether each participant would proceed to explicit action-action training in Phase 7 or would proceed to Session II.

Phase 7: Explicit action-action (equivalence) training. Phase 7 was identical to Phase 6, except that feedback was provided and training continued until participants had reached a mastery training criterion of eight consecutively correct responses. Once again, a multiple baseline design determined whether or not participants would be exposed to explicit equivalence training or whether they would proceed directly to the next session.

\section{Session II}

The procedures outlines in Phases 1 to 7 of Session I were repeated in Session II, but novel stimuli and actions, and their respective names were employed (see Tables 3 \& 4). Specifically, in Session II, the actionobjects relations arms out-B1, touching waist-B1 and arms in-B2, touching neck-B2 were trained and four relations were tested. Specifically, the symmetry object-action relations, B1-touching waist and B2-touching neck were tested in Phase 4 and the equivalence action-action relations touching waist-arms out and touching neck-arms in were tested in Phase 6. Once again, the multiple baseline design determined whether or not participants would be exposed to explicit symmetry and/or equivalence training. Sessions III and IV were identical to the two previous sessions, except that novel stimuli and actions, and their respective names were employed (see Tables 2 and 4).

\section{Results of Part 2}

The data from Part 2 are presented in Table 6. The patterns of responding regarding derived symmetry were similar to those observed in Part 1. Specifically, all participants passed the symmetry test (i.e., derived object-action) without being explicitly trained in symmetry (P2 \& P4 passed on the second test). In contrast, only 1 participant (P4) passed the equivalence test without explicit equivalence action-action training. The other 3 participants demonstrated derived equivalence only after explicit equivalence training. Participant 1 , demonstrated derived equivalence in Session II after explicit action-action training in Phase 7 of Session I. Participant 2 did so in Session III after explicit action-action training in Phase 7 of Session II. Finally, P3 demonstrated derived equivalence in Session IV after explicit action-action training in Phase 7 of Session III. For illustrative purposes, the results from P3 are presented in detail below. 
Table 6

Number of Training Trials Required and Correct Responses During Test Trials for Each Participant in Part 2 of the Experiment

\begin{tabular}{|c|c|c|c|c|}
\hline Participant & 1 & 2 & 3 & 4 \\
\hline \multicolumn{5}{|l|}{ Session I } \\
\hline Phase 1: Action-object training & 8 * & $8^{*}$ & $8^{*}$ & $8^{*}$ \\
\hline Phase 2: Name training & 12 & 12 & 12 & 12 \\
\hline Phase 3: Action-object training & 19 & 18 & 8 & 9 \\
\hline Phase 4: Symm. Test (object-action) & $8 / 8$ & $7 / 8$ & $8 / 8$ & $7 / 8$ \\
\hline Phase 5: Action-object retraining & & ** & & \\
\hline Phase 6: Equiv. Test (action-action) & $6 / \overline{8}$ & $5 / \overline{8}$ & $6 / \overline{8}$ & $5 / \overline{8}$ \\
\hline Phase 7: Action-Action Training & 10 & - & - & - \\
\hline \multicolumn{5}{|l|}{ Session II } \\
\hline Phase 1: Action-object training & 12 & $8^{*}$ & 10 & $8^{*}$ \\
\hline Phase 2: Name training & 12 & 12 & 12 & 12 \\
\hline Phase 3: Action-object training & 18 & 15 & 11 & 8 \\
\hline Phase 4: Symm. Test (object-action) & $8 / 8$ & $8 / 8$ & $8 / 8$ & $8 / 8$ \\
\hline \multicolumn{5}{|l|}{ Phase 5: Action-object retraining } \\
\hline Phase 6: Equiv. Test (action-action) & $8 / \overline{8}$ & $7 / \overline{8}$ & $7 / \overline{8}$ & $8 / \overline{8}$ \\
\hline Phase 7: Action-Action Training & - & 8 & - & - \\
\hline \multicolumn{5}{|l|}{ Session III } \\
\hline Phase 1: Action-object training & _- & 13 & 9 & - \\
\hline Phase 2: Name training & - & 12 & 12 & - \\
\hline Phase 3: Action-object training & - & 11 & 10 & - \\
\hline Phase 4: Symm. Test (object-action) & - & $8 / 8$ & $8 / 8$ & - \\
\hline Phase 5: Action-object retraining & - & & & - \\
\hline Phase 6: Equiv. Test (action-action) & - & $8 / \overline{8}$ & $7 / \overline{8}$ & - \\
\hline Phase 7: Action-Action Training & $\begin{array}{l}- \\
-\end{array}$ & - & 11 & - \\
\hline \multicolumn{5}{|l|}{ Session IV } \\
\hline Phase 1: Action-object training & - & - & 10 & - \\
\hline Phase 2: Name training & - & - & 12 & - \\
\hline Phase 3: Action-object training & - & - & 10 & - \\
\hline Phase 4: Symm. Test (object-action) & - & - & $8 / 8$ & - \\
\hline Phase 5: Action-object retraining & - & - & & - \\
\hline Phase 6: Equiv. Test (action-action) & - & _ & $8 / 8$ & - \\
\hline Phase 7: Action-Action Training & - & - & - & - \\
\hline
\end{tabular}

Note. * indicates that these relations were already trained for these participants in Part I of the experiment.

** indicates that the participant was reexposed to action-object training. In all cases, participant completed the training in eight trials (i.e., the minimum number required).

\section{Participant 3}

In Session I, Participant 3 completed the action-object training in Phase 1 in 8 trials (i.e., wave-A1, clap-A2, see Table 4, Part 2 for the different action-object relations trained and tested in each phase). The name training of two novel actions (i.e., touch forehead \& touch shoulder) was completed in 12 trials. Participant 3 was then exposed to the training of two novel action-object relations (i.e., touch forehead-A1 \& touch shoulder-A2) and completed this training in eight trials. Subsequently, she was exposed to a symmetry object-action test (i.e., A1-touch forehead \& A2-touch shoulder) and produced eight out of eight correct responses. In Phase 6 she was exposed to an equivalence action-action test (i.e., touch 
forehead-wave \& touch shoulder-clap) and failed the test with only six out of eight correct responses. According to the multiple-baseline design, she did not receive explicit action-action equivalence training at this point in the procedure.

In Session II, P3 completed action-object training with different stimuli (arms out-B1 \& arms in-B2, see Table 4) in 10 trials. She received name training with two novel actions (i.e., touch waist \& touch neck) and completed this training in 12 trials. Then she received action-object training with the two novel actions and the same objects and completed this training in 11 trials. Subsequently, she was exposed to a derived object-action symmetry test and passed the test with perfect responding. Finally, she was exposed to an equivalence action-action test and failed with seven out of eight correct responses. Once again, according to the multiple-baseline design, she did not receive explicit action-action equivalence training at this point in the procedure.

In Session III the results were similar to those obtained in Sessions I and II, but new objects and actions (see Table 4) were employed. On this occasion, however, the multiple-baseline design dictated that the child be exposed to explicit action-action training.

Finally, in Session IV, the results were again similar to previous sessions, except that the participant passed the equivalence test after having received explicit action-action equivalence training in the previous session.

In summary, the 4 participants passed all symmetry tests (except P2 and P4 in Session I of Part 2) in both parts of the experiment without necessitating the use of explicit symmetry training. In contrast, only P4 passed the action-action equivalence test without first receiving explicit training in equivalence. For the other 3 participants exemplar equivalence training was necessary to show a derived transformation of functions in accordance with equivalence.

\section{Discussion}

The current study attempted to investigate the existence of repertoires of derived symmetry and equivalence relations with 4 young children and the possible utility of exemplar training when the target repertoires were found to be absent. Part 1 of the study, in particular, attempted to replicate the previous study by Barnes-Holmes et al. (2001a) with respect to the derivation of symmetry relations. In the original study, only 3 of 16 children passed the symmetry test prior to the introduction of explicit symmetry training and no child passed the symmetry test with repeated exposure to the conditional discriminations and the symmetry test alone. In contrast, in the current study, 2 of the 4 children (P1 and P3, both 4:10 years old) passed the first symmetry test and the 2 remaining children (P2 and P4, 4:6 and 4:8 years old, respectively) passed the second test, without explicit symmetry training. One immediate possibility, therefore, is that age primarily distinguished the performances of these two sets of children with regard to the first symmetry test. However, this possibility seems 
unlikely for several reasons. (1) The age difference was very small. (2) All 4 children passed the symmetry test without needing exemplar training. (3) In the original study by Barnes-Holmes et al. (2001a), participants of a same or older age did not pass this same test. As a result, therefore, it seems unlikely that age per se was a critical variable in distinguishing the relative performances of these children on the symmetry testing.

Although the proportion of children who passed the symmetry test immediately in the current study would appear to be discordant with the original study, it is important to emphasize that the original study contained a larger sample of children and may thus be more representative of children of this age group and abilities than the 4 children who participated currently. Furthermore, it is important to note that even those children exposed to explicit symmetry training in the original study required very few exemplars in order to pass the symmetry test. As a result, therefore, one might conclude that for the majority of children of this age and level of verbal ability, there are most likely preexisting repertoires of symmetry relations. Indeed the authors of the original study also noted that "the limited number of exemplars needed in the current study could be taken to indicate that exemplar training was in some way discriminative for an already established behavioral repertoire (p. 304)." The ease with which the four children in the current study generated the target symmetry relations certainly supports this view.

One possible methodological reason for the children's greater ease with symmetry in the current study, relative to the original, concerns the number of name training trials presented to the children. In Part 1 of the present study, the children were required to emit 48 consecutively correct responses, compared to only 24 in the original study. This increased exposure to a bidirectional naming task would likely have facilitated greater ease with symmetry, as observed currently. This possibility is of course consistent with Horne and Lowe's (1996) naming hypothesis, but is also consistent with RFT, which would predict that more exemplars of bidirectional name relations would facilitate stronger bidirectional relations in other domains. Nonetheless, the utility of the naming per se is diminished when one considers the fact that in Experiment 3 of the original study, the children in question were systematically presented with a greater number of naming trials and yet their performances were not better than those observed in the other experiments. Furthermore, when name training was removed in the original study, weaker performances were not recorded. It seems, unlikely, therefore, that the amount of name training per se in the current work was responsible for the greater ease with symmetry observed with the 4 children.

Part 2 of the present study represented a considerable extension of the original work with the investigation of derived combinatorially entailed equivalence relations, as well as mutually entailed relations and an extension of Part 1 of the current work with an investigation of the utility of exemplar training in this regard. Derived equivalence responding emerged only after explicit equivalence training for 3 out of 
the 4 participants. Furthermore, the multiple-baseline design provided some reasonable evidence that the exemplar training was critical to the emergence of these repertoires. Although the current data support the use of explicit exemplar training in the context of combinatorially entailed relations only, the findings largely resemble those from the original study regarding mutually entailed relations. Indeed, once again, only a limited number of explicit training exemplars were required for the emergence of the target relations. Taken together, therefore, both studies suggest that explicit exemplar training may be a useful methodology for facilitating, if not establishing, repertoires of relational responding involving both mutually and combinatorially entailed relations. Perhaps, however, the latter findings are more significant given that RFT would argue that all relational frames require the presence of combinatorially and not just mutually entailed relations. Thus, one might argue that appropriate exemplar training may be useful for facilitating many types of relational responding beyond equivalence or coordination. Indeed, a number of authors have recently reported evidence of this effect when exemplar training appeared to facilitate the establishment ab initio of repertoires of responding in accordance with frames of comparison (i.e., more and less) and opposition in young normally developing children (Barnes-Holmes, Barnes-Holmes, \& Smeets, 2004; Barnes-Holmes, Barnes-Holmes, Smeets, Strand, \& Friman, 2004).

The current work and related studies on the utility of exemplar training on the establishment or facilitation of repertoires of derived relational responding were generated directly by RFT. As well as lending empirical support to the theoretical constructs employed by this account (e.g., mutual and combinatorial entailment), the work on exemplar training indicates that the theory may also make an important contribution to the development of interventions that may prove effective when important relational skills are found to be deficient or absent.

\section{References}

BARNES, D. (1994). Stimulus equivalence and relational frame theory. The Psychological Record, 44, 91-124.

BARNES, D. (1996). Naming as a technical term: Sacrificing behavior analysis at the altar of popularity. Journal of the Experimental Analysis of Behavior, 65, 264-267.

BARNES, D., \& HOLMES, Y. (1991). Radical behaviorism, stimulus equivalence, and human cognition. The Psychological Record, 41, 19-31.

BARNES, D., \& ROCHE, B. (1996). Relational frame theory and stimulus equivalence are fundamentally different: A reply to Saunders' commentary. The Psychological Record, 46, 489-507.

BARNES-HOLMES, Y., BARNES-HOLMES, D., ROCHE, B., \& SMEETS, P. M. (2001a). Exemplar training and a derived transformation of functions in accordance with symmetry. The Psychological Record, 51, 287-308. 
BARNES-HOLMES, Y., BARNES-HOLMES, D., ROCHE, B., \& SMEETS, P. M. (2001b). Exemplar training and a derived transformation of function in accordance with symmetry: II. The Psychological Record, 51, 589-603.

BARNES-HOLMES, Y., BARNES-HOLMES, D., \& SMEETS, P. M. (2004). Establishing relational responding in accordance with opposite as generalized operant behavior in young children. International Journal of Psychology and Psychological Therapy, 4, 559-586.

BARNES-HOLMES, Y., BARNES-HOLMES, D., SMEETS, P. M., STRAND, P., \& FRIMAN, P. (2004). Establishing relational responding in accordance with more-than and less-than as generalized operant behavior in young children. International Journal of Psychology and Psychological Therapy, 4, 531-558.

BOELENS, H. (1994). A traditional account of stimulus equivalence. The Psychological Record, 44, 587-605.

DYMOND, S., \& BARNES, D. (1995). A transformation of self-discrimination response functions through the arbitrarily applicable relations of sameness, more-than, and less-than. Jounal of Experimental Analysis of Behavior, 64, 163-184.

DYMOND, S., \& BARNES, D. (1996). A transformation of self-discrimination response functions in accordance with the arbitrarily applicable relations of sameness and opposition. The Psychological Record, 46, 271-300.

GALIZIO, M. (2003). The abstracted operant: A review of Relational Frame Theory: A Post-Skinnerian Account of Human Language and Cognition, edited by S. C. Hayes, D. Barnes-Holmes, and B. Roche. The Behavior Analyst, 26, 159-169.

GÓMEZ, S., BARNES-HOLMES, D., \& LUCIANO, M. C. (2001). Generalized break equivalence I. The Psychological Record, 51, 131-150.

GÓMEZ, S., BARNES-HOLMES, D., \& LUCIANO, M. C. (2002). Generalized break equivalence II: Contextual control over a generalized pattern of stimulus relations. The Psychological Record, 52, 203-220.

HAYES, S. C. (1991). A relational control theory of stimulus equivalence. In L. J. Hayes \& P. N. Chase (Eds.), Dialogues of verbal behavior (pp.19-40). Reno, NV: Context Press.

HAYES, S. C. (1994). Relational frame theory: A functional approach to verbal events. In S. C Hayes, L. J. Hayes, M. Sato, \& K. Ono (Eds.), Behavior analysis of language and cognition (pp. 9-29). Reno, NV: Context Press.

HAYES, S. C., BARNES-HOLMES, D., \& ROCHE, B. (2001). Relational frame theory. A post-Skinnerian account of human language and cognition. New York. Kluver Academic/Plenum Publishers.

HAYES, S. C., \& HAYES, L. J. (1989). The verbal action of the listener as a basis for rule governance. In S. C. Hayes (Ed.), Rule-governed behavior. Cognition, contingencies and instructional control (pp. 153-190). New York: Plenum.

HAYES, S. C., \& HAYES, L. J. (1992). Verbal relations and the evolution of behavior analysis. American Psychologist, 47, 1383-1395.

HORNE, P. J., \& LOWE, F. (1996). Toward a theory of verbal behaviour. Journal of the Experimental Analysis Behavior, 68, 271-296.

PALMER, D. C. (2004). Data in search of a principle: A review of S. C. Hayes, D. Barnes-Holmes, and B. Roche (Eds.), Relational Frame Theory: A PostSkinnerian Account of Human Language and Cognition. Journal of the Experimental Analysis of Behavior, 81, 189-204.

ROCHE, B., \& BARNES, D. (1996). Arbitrarily applicable relational responding and sexual categorization: A critical test of the derived difference relation. The Psychological Record, 46, 451-475. 
ROCHE, B., \& BARNES, D. (1997). A transformation of respondently conditioned stimulus functions in accordance with arbitrarily applicable relations. Journal of the Experimental Analysis of Behavior, 67, 275-301.

SIDMAN, M. (1994). Equivalence relations and behavior: A research story. Boston: Authors Cooperative.

SMEETS, P. M., BARNES, D., \& LUCIANO, M. C. (1995). Reversal of emergent simple discrimination in children: A component analysis. Journal of Experimental Child Psychology, 60, 327-343.

STEELE, D., \& HAYES, S. C. (1991). Stimulus equivalence and arbitrarily applicable relational responding. Journal of the Experimental Analysis of Behavior, 56, 519-555.

WULFERT, E., \& HAYES, S. C. (1988). The transfer of conditional sequencing through conditional equivalence classes. Journal of the Experimental Analysis of Behavior, 50, 125-144. 
GÓMEZ ET AL. 